\title{
Silent myocardial infarction and risk of heart failure
}

\author{
Timir K. Paul ${ }^{1}$, Debabrata Mukherjee ${ }^{2}$ \\ ${ }^{1}$ Division of Cardiology, East Tennessee State University, Johnson City, TN, USA; ${ }^{2}$ Division of Cardiovascular Medicine, Texas Tech University \\ Health Sciences Center, El Paso, Texas, USA \\ Correspondence to: Debabrata Mukherjee, MD, MS. Division of Cardiovascular Medicine, Texas Tech University Health Sciences Center, El Paso, \\ Texas 79905, USA. Email: debabrata.mukherjee@ttuhsc.edu. \\ Comment on: Qureshi WT, Zhang ZM, Chang PP, et al. Silent Myocardial Infarction and Long-Term Risk of Heart Failure: The ARIC Study. J Am \\ Coll Cardiol 2018;71:1-8.
}

Submitted Sep 10, 2018. Accepted for publication Sep 17, 2018.

doi: 10.21037/atm.2018.09.45

View this article at: http://dx.doi.org/10.21037/atm.2018.09.45

Heart failure (HF) is a major public health problem with a current estimate of 6.5 million Americans of more than 20 years of age having a diagnosis of HF (1). This is an increase from 5.7 million US adults with HF based on estimates from the National Health and Nutrition Examination Survey (NHANES) conducted 2009 to 2012 (1). Furthermore, epidemiological projections suggest that HF prevalence will increase $46 \%$ in the next two decades, resulting in more than 8 million adults with $\mathrm{HF}$ in the US (2). The National Heart, Lung, and Blood Institute (NHLBI)-sponsored Chicago Heart Association Detection Project in Industry (CHA), Atherosclerosis Risk in Communities (ARIC), and Cardiovascular Health Study (CHS) data reported that HF incidence is close to 21 per 1,000 population after 65 years of age and is likely to be a particular issue going forward among our aging population (3). HF is also a common problem throughout the world with estimates of prevalence as high as $6.7 \%$ in some parts of the world (1).

To combat/prevent HF, it is important to understand the risk or predisposing factors for HF. It should be noted that risk factors for HF vary substantially across different regions of the world, with hypertension having strong association with HF in Latin America, Eastern Europe, the Caribbean, and sub-Saharan Africa (4). On the other hand, ischemic heart disease prevalence among HF patients is highest among Europeans and North Americans and is a key risk/predisposing factor in these regions (4). The Olmstead County, MN, data indicate that ischemic heart disease, hypertension, diabetes mellitus, obesity, and smoking are responsible for more than $50 \%$ of HF cases in their population (5). The odds ratios (ORs) or relative risk (RRs) and the population attributable risks (PARs) for the different risk factors are as follows: ischemic heart disease OR, 3.1 and overall PAR, 20\% (higher in males, 23\% versus $16 \%$ in females); cigarette smoking RR, 1.4 and PAR, $14 \%$; hypertension RR, 1.4 and PAR, 20\% (higher in females, $28 \%$ versus $13 \%$ in males); obesity RR, 2.0 and PAR, $12 \%$; diabetes mellitus OR, 2.7 and PAR, 12\%; dietary sodium intake RR, 1.4 and PAR, not available; and valvular heart disease RR, 1.5 and PAR, $2 \%$ with ischemic heart disease having the strongest association with HF (6). The risk of HF with a known history of prior myocardial infarction (MI) is well established (7) but the role of prior undiagnosed or silent MI remains poorly defined. The issue is particularly important and challenging as silent MI may account for approximately half of the total number of MIs. Since prior MI or ischemic heart disease remains a key predisposing factor for HF, it is crucial that we diagnose these silent MIs prior to development of HF. There is also certainly a need to better define the risk of HF with silent MI as well as possible strategies to diagnose silent MI.

To this end, a recent study analyzed 9,243 participants from the ARIC study who were free of cardiovascular disease at baseline to examine the association of silent MI and clinically manifested MI with HF, as compared with patients with no prior MI (8). The incidence rate of HF was higher in both clinically manifested MI and silent MI compared to no prior MI (incidence rate per 1,000 personyears was $30.4,16.2$, and 7.8 , respectively; $\mathrm{P}$ value $<0.001$ ). Multivariable adjusted Cox proportional hazard models also demonstrated both clinically manifested MI and silent MI, 
compared to no MI, were significantly associated with HF (incidence rate per 1,000 person-years was 2.85 and 1.35 respectively) (8). This study underscores the importance of silent/undiagnosed MI as an important risk factor for development of future HF.

The next important clinical question is whether screening for silent MI with tests is cost-effective and whether preventative therapies in patients with silent MI would be beneficial in reducing the risk of future HF. In the ARIC study, silent MI was defined as electrocardiographic (ECG) evidence of new MI at subsequent visits that was not present at the first visit. The presence of $\mathrm{Q}$ waves in an ECG has been used to diagnose prior MI for decades. One study assessed the how accurate an ECG is in detecting prior MI compared with cardiac magnetic resonance imaging (MRI) and reported a very modest sensitivity of $48.4 \%$; specificity of $83.5 \%$; a positive predictive accuracy of $72.0 \%$; and a negative predictive accuracy of $64.2 \%$ with ECG. Sensitivity was reasonably high with large MI (64\%), but specificity declined to $72 \%$ (9). These data would appear to suggest that the poor sensitivity and the modest negative predictive value of ECG criteria may seriously curb its accuracy for diagnosis of prior MI. Furthermore, the presence of a right bundle branch block (RBBB) while typically considered to interfere with the ECG diagnosis of prior MI, recent clinical studies have reported association between RBBB and both false-positive as well as falsenegative ECG diagnoses of MI (10). Hence, the finding of a new $\mathrm{Q}$ wave in an asymptomatic individual with a RBBB pattern on ECG needs additional testing to confirm the diagnosis of prior MI. Similarly, left anterior fascicular block may also confound the diagnosis of prior MI based solely on Q waves (11). Based on these and other evidence, an abnormal ECG may not be necessarily diagnostic of a prior silent MI.

To confirm silent $\mathrm{MI}$ in a patient with Q waves on ECG, the first step should be to repeat another ECG to rule out lead malposition as a cause of abnormal ECG. If repeat ECG confirms evidence of prior MI, several imaging tests may be considered to confirm MI or presence of significant coronary artery disease such as echocardiography, radionuclide ventriculography, myocardial perfusion scintigraphy, cardiac/coronary computed tomography (CT) angiography and cardiac MRI. Freeman et al. assessed the ability of radionuclide ventriculography and echocardiography to assess regional left ventricular wall motion in patients with healed prior MI (12). They reported a sensitivity in detecting wall motion abnormalities of $83 \%$ for echocardiography and $77 \%$ for radionuclide ventriculography in the anterolateral area and apical (95\%, and $84 \%$ respectively) segment and least for the inferior segment (48\%, 48\%) (12). Specificity of echocardiography and radionuclide ventriculography was quite good, ranging from $94 \%$ in the anterolateral myocardial wall to $71 \%$ in the septal myocardial wall for echocardiography, and from $91 \%$ in the inferior wall to $81 \%$ in the posterobasal and septal wall for radionuclide ventriculography (12). Nikolaou et al. examined the diagnostic accuracy of multidetector cardiac CT for detecting significant coronary heart disease in patients with known coronary heart disease and no prior coronary heart disease on both per patient and per segment analyses (13). In this study, multidetector cardiac CT showed a sensitivity of $82 \%$ and $86 \%$, respectively for the identification of stenoses of $>50 \%$ and $>75 \%$ per segment, and specificity and negative predictive value were as high as $95 \%$ and $97 \%$, respectively.

Cardiac MRI offers high spatial resolution and, in contrast to other imaging modalities mentioned above, has better ability to discern myocardial fibrosis and distribution of infarct in the myocardium and across different myocardial layers. One study reported a sensitivity of contrastenhanced Cardiac MRI for the detection of prior MI was $91 \%$ and a specificity of $100 \%$ (14). Another study to assess the diagnostic precision of CMR to detect coronary heart disease reported a sensitivity of CMR of $86.5 \%$ (95\% CI, $81-90 \%$ ), specificity of $83.4 \%$ (95\% CI, 79-86\%), a positive predictive value of $77 \%$ (95\% CI, $72-81 \%$ ), and negative predictive value of $90.5 \%$ (95\% CI, 87.1-93.0\%) (15). Imaging modalities for the diagnosis of prior $\mathrm{MI}$ and significant coronary artery disease are summarized in Table 1 . The choice of subsequent testing to confirm MI in those with abnormal ECG will depend on several factors such as local expertise, availability, renal function and patient and physician preference.

It is obvious that the diagnosis of previous silent MI is clinically important as it is an important predisposing factor for $\mathrm{HF}$ and overall cardiovascular morbidity and mortality (16). Once a prior MI is detected/confirmed on testing, early initiation of therapies may potentially prevent overt HF and may improve the patient's long-term prognosis.

Of note, the United States Preventive Service Task Force currently recommends against screening with ECG for the prognostication of cardiac events in asymptomatic individuals who are at low risk for such events and concludes that the current evidence is not sufficient to 
Table 1 Imaging modalities for the diagnosis of prior myocardial infarction and significant coronary artery disease

\begin{tabular}{lcccc}
\hline Modality & Sensitivity (\%) & Specificity (\%) & Positive predictive value (\%) & Negative predictive value (\%) \\
\hline Electrocardiography & 48.4 & 83.5 & 72 & 64.2 \\
Echocardiography & $48-95$ & $71-94$ & NA & NA \\
Radionuclide ventriculography & $48-84$ & $81-91$ & NA & NA \\
Cardiac CT & $82-86$ & 97 & NA & 97 \\
Cardiac MRI & 91 & 100 & 77.2 & 90.5 \\
\hline
\end{tabular}

NA, not available; CT, computerized tomography; MRI, magnetic resonance imaging.

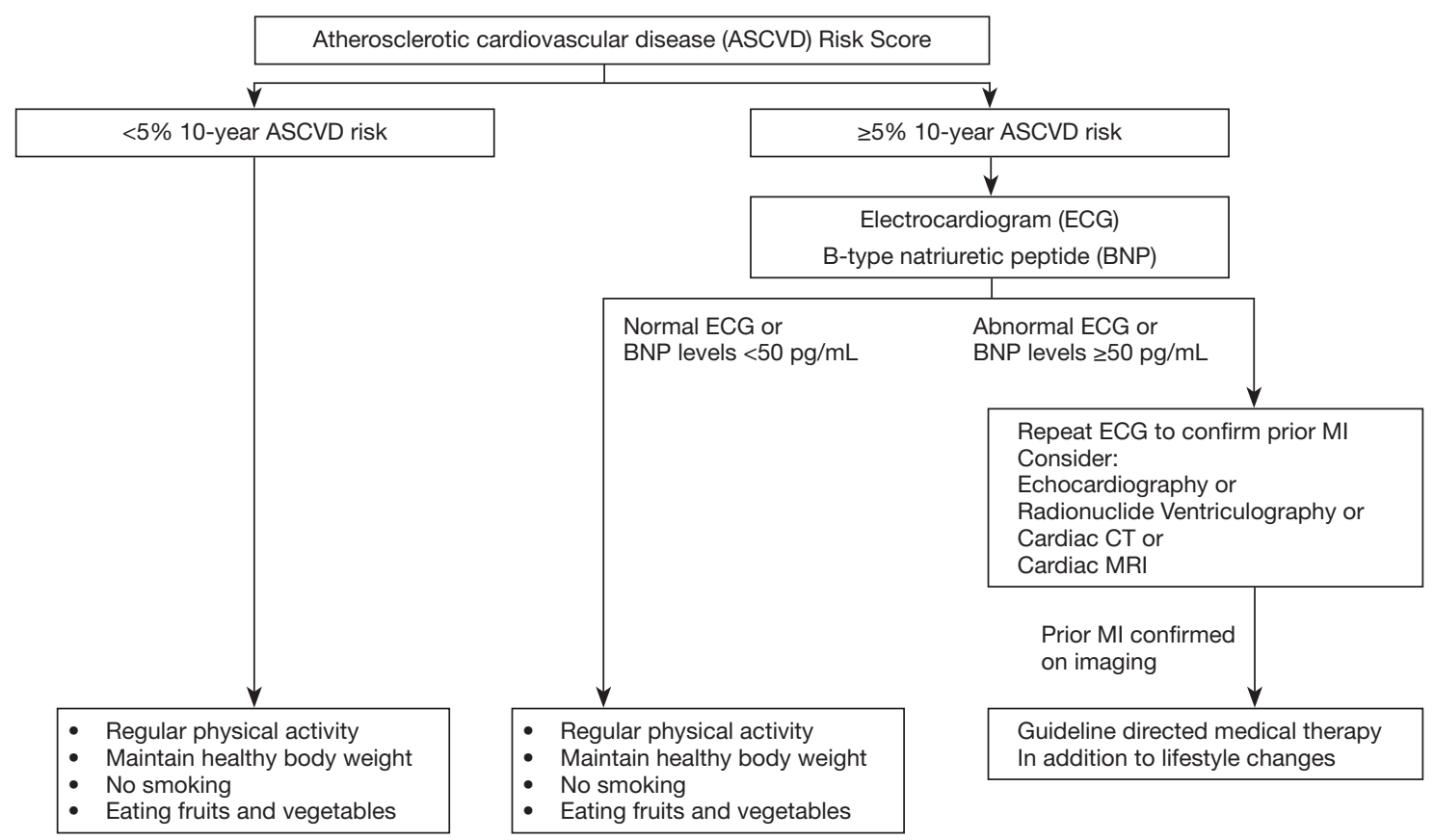

Figure 1 Simplified algorithm for identification and management of individuals at high risk of heart failure. ASCVD, atherosclerotic cardiovascular disease; MI, myocardial infarction; CT, computerized tomography; MRI, magnetic resonance imaging.

appraise benefits and risks of screening with ECG for the prediction of cardiovascular events in asymptomatic individuals at intermediate or high risk given lack of benefit and potential for harm as a result of unnecessary followup tests and interventions (17). The 2017 Focused Update recommends utilizing B-type natriuretic peptide biomarkerbased testing for individuals at perceived risk of developing HF, followed by multidisciplinary care including a cardiovascular specialist directing guideline evidence-based medical therapy, to prevent the evolution of left ventricular dysfunction (systolic or diastolic) and/or incipient HF (18). Judicious use of B-type natriuretic peptide biomarker screening for those at risk for HF and use of ECG in those at least at intermediate or at high risk seems like a reasonable approach to prevent this life-threatening condition. Finally, in addition to pharmacotherapies such as angiotensin converting enzyme inhibitors, angiotensin receptor blockers, aldosterone antagonists (19) and betablockers (20), lifestyle modifications such as regular physical exercise, maintaining a healthy body weight, not smoking, eating fruits and vegetables also will help to reduce risk of HF and should be recommended to all individuals at risk for ischemic heart disease and HF. Figure 1 provides a simplified algorithm for clinicians to use ECG and B-type natriuretic peptide levels to selectively identify individuals at high risk for HF using the atherosclerotic cardiovascular disease (ASCVD) risk score and appropriately manage those with prior $\mathrm{MI}$ or elevated biomarkers. 


\section{Acknowledgements}

None.

\section{Footnote}

Conflicts of Interest: The authors have no conflicts of interest to declare.

\section{References}

1. Benjamin EJ, Virani SS, Callaway CW, et al. Heart Disease and Stroke Statistics-2018 Update: A Report From the American Heart Association. Circulation 2018;137:e67-492.

2. Heidenreich PA, Albert NM, Allen LA, et al. Forecasting the impact of heart failure in the United States: a policy statement from the American Heart Association. Circ Heart Fail 2013;6:606-19.

3. Huffman MD, Berry JD, Ning H, et al. Lifetime risk for heart failure among white and black Americans: cardiovascular lifetime risk pooling project. J Am Coll Cardiol 2013;61:1510-7.

4. Khatibzadeh S, Farzadfar F, Oliver J, et al. Worldwide risk factors for heart failure: a systematic review and pooled analysis. Int J Cardiol 2013;168:1186-94.

5. Dunlay SM, Weston SA, Jacobsen SJ, et al. Risk factors for heart failure: a population-based case-control study. Am J Med 2009;122:1023-8.

6. Nkomo VT, Gardin JM, Skelton TN, et al. Burden of valvular heart diseases: a population-based study. Lancet 2006;368:1005-11.

7. Weir RA, McMurray JJ. Epidemiology of heart failure and left ventricular dysfunction after acute myocardial infarction. Curr Heart Fail Rep 2006;3:175-80.

8. Qureshi WT, Zhang ZM, Chang PP, et al. Silent Myocardial Infarction and Long-Term Risk of Heart Failure: The ARIC Study. J Am Coll Cardiol 2018;71:1-8.

9. Asch FM, Shah S, Rattin C, et al. Lack of sensitivity of the electrocardiogram for detection of old myocardial infarction: a cardiac magnetic resonance imaging study. Am Heart J 2006;152:742-8.

10. Gussak I, Wright RS, Bjerregaard P, et al. False-negative and false-positive ECG diagnoses of $\mathrm{Q}$ wave myocardial infarction in the presence of right bundle-branch block. Cardiology 2000;94:165-72.

11. Shettigar UR, Pannuri A, Barbier GH, et al. Significance of anterior Q waves in left anterior fascicular block-a clinical and noninvasive assessment. Clin Cardiol 2002;25:19-22.

12. Freeman AP, Giles RW, Walsh WF, et al. Regional left ventricular wall motion assessment: comparison of two-dimensional echocardiography and radionuclide angiography with contrast angiography in healed myocardial infarction. Am J Cardiol 1985;56:8-12.

13. Nikolaou K, Knez A, Rist C, et al. Accuracy of 64-MDCT in the diagnosis of ischemic heart disease. AJR Am J Roentgenol 2006;187:111-7.

14. Wu E, Judd RM, Vargas JD, et al. Visualisation of presence, location, and transmural extent of healed $\mathrm{Q}$-wave and non-Q-wave myocardial infarction. Lancet 2001;357:21-8.

15. Greenwood JP, Maredia N, Younger JF, et al. Cardiovascular magnetic resonance and single-photon emission computed tomography for diagnosis of coronary heart disease (CE-MARC): a prospective trial. Lancet 2012;379:453-60.

16. Law MR, Watt HC, Wald NJ. The underlying risk of death after myocardial infarction in the absence of treatment. Arch Intern Med 2002;162:2405-10.

17. Moyer VA; U.S. Preventive Services Task Force. Screening for coronary heart disease with electrocardiography: U.S. Preventive Services Task Force recommendation statement. Ann Intern Med 2012;157:512-8.

18. Yancy CW, Jessup M, Bozkurt B, et al. 2017 ACC/ AHA/HFSA Focused Update of the 2013 ACCF/AHA Guideline for the Management of Heart Failure: A Report of the American College of Cardiology/American Heart Association Task Force on Clinical Practice Guidelines and the Heart Failure Society of America. Circulation 2017;136:e137-61.

19. Chatterjee S, Moeller C, Shah N, et al. Eplerenone is not superior to older and less expensive aldosterone antagonists. Am J Med 2012;125:817-25.

20. Chatterjee S, Biondi-Zoccai G, Abbate A, et al. Benefits of beta blockers in patients with heart failure and reduced ejection fraction: network meta-analysis. BMJ 2013;346:55 .

Cite this article as: Paul TK, Mukherjee D. Silent myocardial infarction and risk of heart failure. Ann Transl Med 2018;6(Suppl 1):S35. doi: 10.21037/atm.2018.09.45 